\title{
Control Theoretic Properties of Physiological Controller Motifs
}

\author{
Kristian Thorsen*, Peter Ruoff ${ }^{\dagger}$ and Tormod Drengstig* \\ * Department of Electrical Engineering and Computer Science, University of Stavanger, Norway. Email: kristian.thorsen@uis.no \\ †Centre for Organelle Research, University of Stavanger, Norway.
}

\begin{abstract}
Identifying biophysical mechanisms that provide regulation and control is essential for our understanding of living systems. However, the distance between life sciences and control theory can be a challenge. Here, we describe, and show the control theoretic properties of a set of biochemical reaction schemes, so-called controller motifs. These controller motifs have similarities with industrial control systems, and have properties such as setpoints, integral gain, and setpoint weight. Once identified, a system understanding of such mechanisms can help synthetic biologists in selecting suitable targets to alter in construction of new biological systems. From a control theoretic viewpoint we identify which biochemical rate constant or property affect the setpoint and the dynamic response of a biophysical controller motif. We also show how a biological system consisting of two antagonistic regulatory mechanisms can be compared to a control engineering problem of controlling the water level in a tank. The similarity between biological systems and control engineering provides theoretical insight, and clears the way to an engineer's approach to synthetic biology.
\end{abstract}

\section{INTRODUCTION}

The ability of living systems to adjust to environmental changes or disturbances are often compared and linked to control theory [1]-[4]. The biological and physiological concepts of robustness [5]-[7], homeostasis [8]-[10], and adaptation [11]-[13] are closely related to the control theoretic properties of setpoint regulation and disturbance rejection [14].

A typical observation from biological systems is shown in Fig. 1, a step-based disturbance and the corresponding response in the output. An example of such behavior is the change of firing rate of a single optic nerve cell (ommatidium) from the compound eye of the horseshoe crab upon light perturbations [11], [15].

We have recently presented a set of two-component molecular controller motifs [16], which are reaction kinetic schemes of how two chemical species can interact to achieve robust adaptation and homeostasis. These controller motifs show how the synthesis and degradation of two chemical species, controller species $(E)$ and controlled species $(A)$, can interact to achieve robust control of the concentration of $A$, see Fig. 2. In control theoretic terms $A$ is called the controlled variable $(\mathrm{CV})$ and $E$ is the manipulated variable (MV).

Uncontrolled disturbances in inflow and outflow of $A$, marked $d_{i}$ and $d_{o}$, are compensated for by $E$ which adjusts the compensatory flux $j$ so that $A$ is controlled to its setpoint (homeostatic) value. This adjustment of the compensatory flux happens either by activation (increasing $E$ increases $j$ ) or by

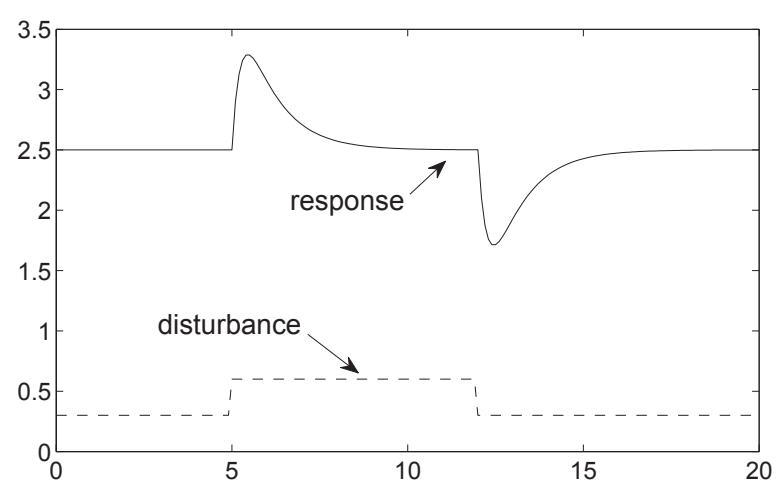

Fig. 1. A typical experimental disturbance step response from living organisms.

inhibition (increasing $E$ decreases $j$ ), marked with plus and minus signs respectively.

There are four configurations by which $E$ can affect $A$ through the compensatory flux $j$, these are:

1) Synthesis of $A$ is:

a) Activated by $E$ (inflow 1 and 3 ).

b) Inhibited by $E$ (inflow 2 and 4 ).

2) Degradation of $A$ is:

a) Activated by $E$ (outflow 5 and 7).

b) Inhibited by $E$ (outflow 6 and 8 ).

Likewise there are four configurations by which the controller system $(E)$ can be affected by $A$ through the measurement flux $m$.

Controller motifs can be identified to exist in cells and organisms; the controller species $(E)$ is typically an enzyme, a hormone, or a transporter protein, and the controlled species $(A)$ is typically a vital chemical substance that has to be maintained within a defined range to secure survivability. One example is IRT1 a transporter protein $(E)$ which controls the uptake of iron $(A)$ in plants [16], [17]. Another example is parathyroid hormone $\left(E_{1}\right)$ and calcitonin hormone $\left(E_{2}\right)$, found in many animals including mammals, which controls the concentration of calcium in blood $(A)$ [10], [18], [19]. Controller motifs have also been used in practical applications in synthetic biology. Control structures have been artificially implemented and used to create adapting gene networks [20] and mechanisms that are able to prevent lethal disease outcomes from virus infections in bacterial cells [21]. 


\section{inflow controllers}
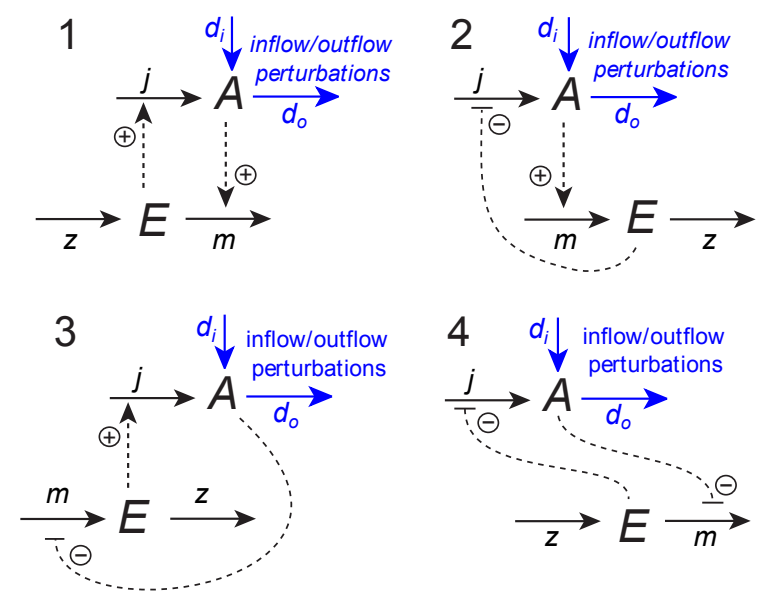

\section{outflow controllers}

\section{5}

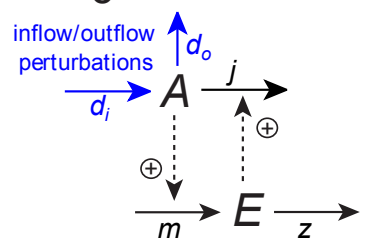

7

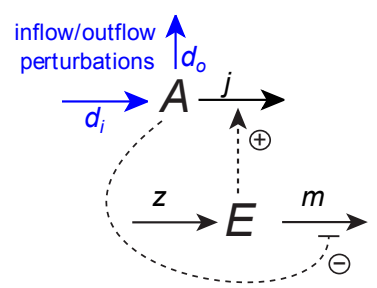

6

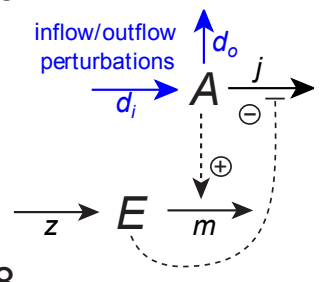

8

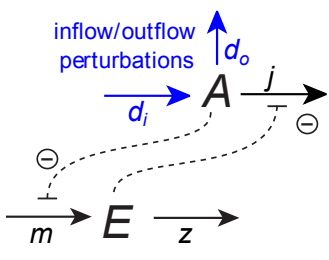

Fig. 2. Two-component molecular controller motifs. The motifs are divided into two subgroups: i) inflow controllers where the controller species (E) affects the inflow of the controlled species $(A)$, and ii) outflow controllers where the controller species $(E)$ affects the outflow of the controlled species $(A)$. The uncontrolled inflow and outflow of $A$, marked $d_{i}$ and $d_{o}$, is compensated for by the compensatory flux $j$. $E$ receives information about the level of $A$ through the measurement flux $m, z$ is the inflow or outflow of $E$ that is unaffected by $A$.

We became fascinated by the fact that the molecular controller motifs show a dynamic response behavior close to that of standard industrial controllers such as the PID controller. In this paper we explore the control theoretic properties of these molecular controller motifs in a control theoretic (mathematical) framework. We show that the controller motifs have properties that are familiar to industrial controllers such as setpoint and integral gain, but also that they have more unusual properties such as nonlinear measurement functions and setpoint weight.

\section{CONTROL THEORETIC PROPERTIES}

The kinetics of the compensatory flux $j$ with respect to $E$ can take different forms depending on the biochemical reaction mechanism. Not all enzymes have the same reaction mechanism, and even apparently simple reactions may be a series of different subreactions, each with its own kinetics.

For the sake of simplicity we will here formulate compensatory fluxes that are activated by $E$ as first-order kinetics with respect to $E$. Compensatory fluxes that are that are inhibited by $E$ are described by the uncompetitive (catalytic) inhibition type kinetics where $E$ affects the maximum reaction rate [22].

Definition 1: Compensatory flux.

The compensatory flux $j$ has the form:

$$
\begin{aligned}
j(\cdot, E) & =f_{c} f(\cdot) E, \quad \text { activation. } \\
j(\cdot, E) & =f_{c} f(\cdot) \frac{K_{i}^{E}}{K_{i}^{E}+E}, \quad \text { inhibition. }
\end{aligned}
$$

$f_{c}$ is a constant and $f(\cdot)$ is a function describing the flux dependence on other parameters (e.g. level of $A$, temperature, or $\mathrm{pH}$ ), and $K_{i}^{E}$ is the inhibition constant.

Treating $A$ as the state variable we can set up the differential equation describing the system which is to be controlled:

$$
\dot{A}=d_{i}(\cdot)-d_{o}(\cdot) \pm j(\cdot, E)
$$

The compensatory flux is added in inflow motifs, and substracted in outflow motifs.

To set up an expression for the controller $E$ we will first define the measurement flux $m$, that is the flux by which information about the amount of $A$ is coupled to the inflow or outflow of $E$. (How the controller gets information about the state of the system.) Likewise as for the compensatory flux the measurement flux can take different forms dependent on the biochemical reaction mechanism. Using the same forms for activation and inhibition as above we define:

Definition 2: Measurement flux.

The measurement flux $m$ can take two forms:

$$
\begin{aligned}
& m(\cdot, E)=g_{c} g(\cdot) A, \quad \text { activation } \\
& m(\cdot, E)=g_{c} g(\cdot) \frac{K_{i}^{A}}{K_{i}^{A}+A}, \quad \text { inhibition. }
\end{aligned}
$$

$g_{c}$ is a constant and $g(\cdot)$ is a function describing the flux dependence on other parameters (e.g. level of $E$, temperature, or $\mathrm{pH}$ ), and $K_{i}^{A}$ is the inhibition constant.

Without loss of generality we will also write the z-flux on the form $z_{c} z(\cdot)$, where $z_{c}$ is a constant, and $z(\cdot)$ is a function describing the flux dependence on other parameters (e.g. level of $E$, temperature, or $\mathrm{pH}$ ).

\section{A. Controller motifs 1 and 6}

For motif 1 and 6 the differential equation describing the changes in $E$ is:

$$
\dot{E}=z_{c} z(\cdot)-g_{c} g(\cdot) A
$$

For the simple case when $g(\cdot)$ and $z(\cdot)$ are 1 the setpoint for controller motif 1 and 6 is (setting $\dot{E}=0$ ):

$$
A_{\text {set }}=\frac{z_{c}}{g_{c}}
$$


Rearranging (6) gives:

$$
\dot{E}=g_{c}\left[z(\cdot) \frac{z_{c}}{g_{c}}-g(\cdot) A\right]
$$

where (8) is structurally similar to the standard integral control law from control engineering [23], i.e. $\dot{u}=G_{i}(r-y)$ where $r$ is the reference (setpoint), $y$ is the measurement and $G_{i}$ is the integral gain. In (8) $g_{c}$ acts as the integral gain, and is at the same time part of the setpoint. This means that an increased integral gain must be accompanied by an increase in the $z_{c}$ parameter to keep the setpoint unchanged.

The $z(\cdot)$ function acts as a way to move the setpoint from its basal value as given in (7). In this respect we can say that $z(\cdot)$ acts as a setpoint weight.

If the measurement function $g(\cdot)$ is different from 1 we do not have a direct measure of $A$. In organisms and cells the function $g(\cdot)$ may reflect the overall signal-transduction path by which information about the concentration of $A$ is transmitted to the controller.

\section{B. Controller motifs 2 and 5}

For motif 2 and 5 the differential equation describing the changes in $E$ is:

$$
\dot{E}=g_{c} g(\cdot) A-z_{c} z(\cdot)
$$

For the simple case when $g(\cdot)$ and $z(\cdot)$ are 1 the setpoint for controller motif 2 and 5 is:

$$
A_{\text {set }}=\frac{z_{c}}{g_{c}}
$$

Rearranging (9) gives:

$$
\dot{E}=-g_{c}\left[z(\cdot) \frac{z_{c}}{g_{c}}-g(\cdot) A\right]
$$

These two controller motifs are structurally (11) equal to controller motifs 1 and 6 (7) except for the negative integral gain $G_{i}=-g_{c}$. This is necessary as controller motif 2 inhibits the inflow of $A$ while motif 1 activates the inflow of $A$, see Fig. 2. To increase the inflow of $A$, through the compensatory flux $j$, controller motif 2 must decrease $E$ (negative gain) instead of increasing it as motif 1 does. Likewise motif 5 must decrease $E$ to increase $A$ (negative gain), as this will decrease the compensatory flux and reduce the total outflow of $A$.

\section{Controller motifs 3 and 8}

For motif 3 and 8 the differential equation describing the changes in $E$ is:

$$
\dot{E}=g_{c} g(\cdot) \frac{K_{i}^{A}}{A+K_{i}^{A}}-z_{c} z(\cdot)
$$

For the simple case when $g(\cdot)$ and $z(\cdot)$ are 1 the setpoint for controller motif 2 and 8 is:

$$
A_{\text {set }}=\frac{g_{c} K_{i}^{A}}{z_{c}}-K_{i}^{A}
$$

Rearranging (12) gives:

$$
\begin{array}{r}
\dot{E}=\frac{z_{c} z(\cdot) g(\cdot)}{A+K_{i}^{A}}\left[\frac{1}{z(\cdot)}\left(\frac{g_{c} K_{i}^{A}}{z_{c}}-K_{i}^{A}\right)\right. \\
\left.-\left(\frac{1}{g(\cdot)}\left(A+K_{i}^{A}\right)-\frac{K_{i}^{A}}{z(\cdot)}\right)\right]
\end{array}
$$

The inhibition leads to a more complicated expression, but the structure is still similar to the standard integral control law. This can be seen more easily considering the simple case when $g(\cdot)$ and $z(\cdot)$ are 1 , and (14) reduces to

$$
\dot{E}=\underbrace{\frac{z_{c}}{A+K_{i}^{A}}}_{G_{i}}[\underbrace{\left(\frac{g_{c} K_{i}^{A}}{z_{c}}-K_{i}^{A}\right)}_{A_{\text {set }}}-A]
$$

where the bracketed value is the difference between the setpoint and the actual $A$ value.

For controller motifs 3 and 8 the integral gain $G_{i}$ is dependent on $A$, the state that is controlled. Seen in control theoretical terms this is a sort of gain scheduling [23]. The gain reduces as $A$ increases, indicating that a higher gain might be preferential at low concentrations of $A$ where it will lead to a faster response. The reduction in gain as $A$ rises is slowing the response so that controller induced overshoot into harmful high concentrations of $A$ are less likely, a property that might increase cell and organism survivability.

The more complicated behavior of controller motif 3 and 8 is shown in how $g(\cdot)$ and $z(\cdot)$ interact with the control law. The function $z(\cdot)$ is for these motifs not just a setpoint weight; it affects the setpoint, the integral gain and the measurement of $A$. The integral gain is also dependent on the $g(\cdot)$ function.

\section{Controller motifs 4 and 7}

These two controller motifs are in mathematical structure equal to controller motifs 3 and 8 (14) except for the negative integral gain. This is the same as seen between the motif pairs 2,5 and 1,6 .

\section{TIME AND FREQUENCY RESPONSE}

We use inflow motif 3 to illustrate typical responses to step changes in the setpoint and disturbance (we do only consider motif 3 in this part due to space limitation and to avoid repetition). The response is primarily dependent on the integral gain parameter $G_{i}$. In the analysis we have set the inflow disturbance $d_{i}$ to zero and the outflow disturbance $d_{o}$ is on the form $d_{c} A$. In addition we have used the following parameters: $f_{c}=K_{i}^{A}=d_{c}=1, z_{c}=\{0.5,5,50\}, g_{c}=\{2.5,25,250\}$, and $z(\cdot)=g(\cdot)=f(\cdot)=1$. The three different $z_{c}$ and $g_{c}$ values gives three different gain settings $\left(G_{i}=\{0.1,1,10\}\right)$.

Fig. 3 shows how inflow controller 3 reacts to a $25 \%$ change in its setpoint value for these gain settings. The setpoint step is made by perturbing $g_{c}$ (changing the setpoint without changing the gain). Since the gain has the form $G_{i}=z_{c} /\left(A+K_{i}^{A}\right)$ the gain reduces when $A$ increases, so that $G_{i}=\{0.1,1,10\}$ for $A=4$ changes to $G_{i} \approx\{0.083,0.83,8.3\}$ when $A=5$.

Perturbing the disturbance to create a step change gives the response shown in Fig. 4. 


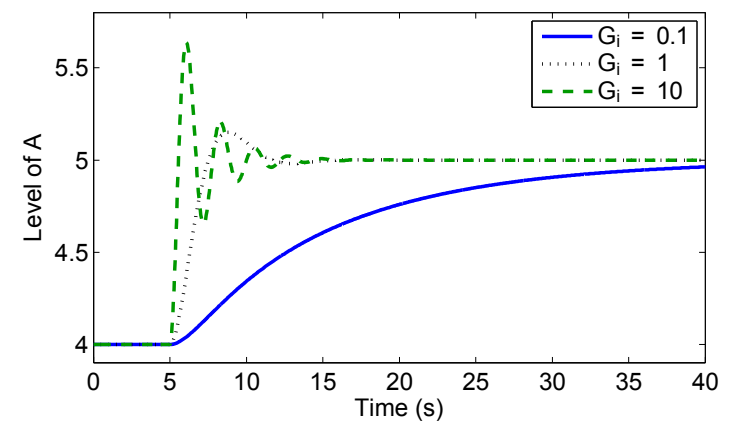

Fig. 3. Response to a step change in the setpoint for inflow controller motif 3. At time $t=5$ the setpoint is changed from 4 to 5 by perturbing $g_{c}$. The response is shown for three different gain settings, $G_{i}=0.1$ (solid blue), $G_{i}=1$ (dotted black), and $G_{i}=10$ (dashed green).

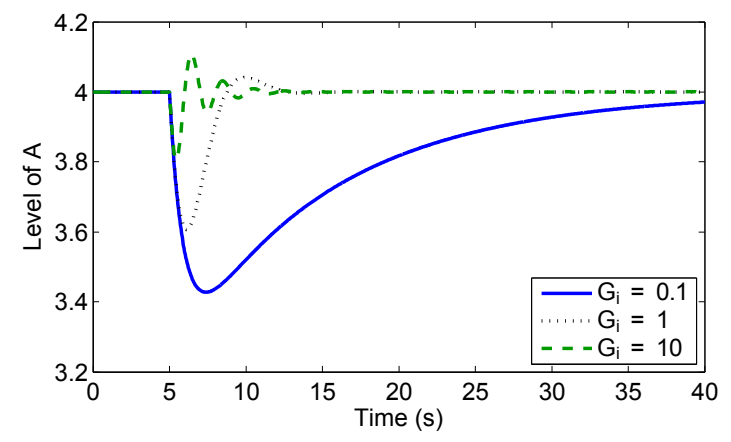

Fig. 4. Response to a step change in the outflow disturbance for inflow controller motif 3. At time $t=5$ the disturbance is changed from $d_{c}=1$ to $d_{c}=1.2$. The response is shown for three different gain settings, $G_{i}=0.1$ (solid blue), $G_{i}=1$ (dotted black), and $G_{i}=10$ (dashed green).

The response time of the controller motif is shorter with high gains, but at the cost of overshoot and oscillations (Figs. 3 and 4). The response time and overshoot properties of a biological system, with controller motifs as presented here, are a reflection of reaction kinetic rates and constants, much more than a reflection of any particular control structure.

To show the frequency response we have calculated the closed loop transfer function [24], [25] of inflow controller motif 3. Since the motif equations are nonlinear they have to be linearized before finding the transfer function, which thus is only valid in some region near to the setpoint. The closed loop transfer function from the reference $A_{\text {set }}$ to the output $A$ is:

$$
M(s)=\frac{z_{c}^{2} f_{c}}{g_{c} K_{i}^{A} s^{2}+g_{c} K_{i}^{A} d_{c} s+z_{c}^{2} f_{c}}
$$

and is plotted in Fig. 5. The bandwidth of the controller is as expected increased when the integral gain is increased. Nevertheless, as shown in the step responses (Figs. 3 and 4), high gain leads to overshoot, also indicated by the resonance top in Fig. 5.

\section{MANipulating THE CONTROLleR}

Although not the main point of this paper, we will here briefly discuss how some aspects from this treatment can be used in practical applications. We use motif 3 to illustrate how

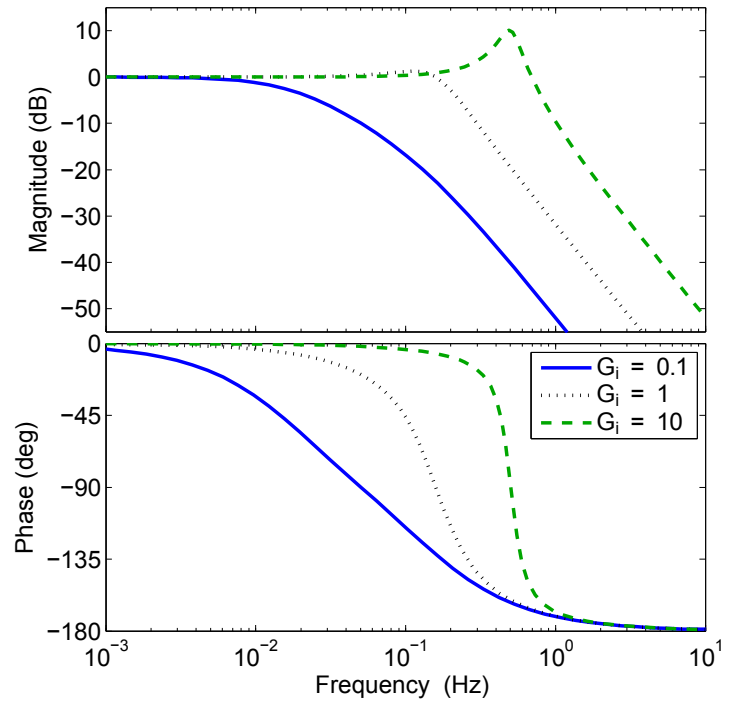

Fig. 5. Close loop frequency response for inflow controller motif 3. The response is shown for three different gain settings, $G_{i}=0.1$ (solid blue), $G_{i}=1$ (dotted black), and $G_{i}=10$ (dashed green).

our results can help synthetic biologist identifying suitable targets in order to manipulate properties such as the setpoint and the dynamic response.

To change the setpoint of motif 3 , shown in (13), we can try to alter either the rate of the measurement flux $g_{c}$, the rate of the z-flux $z_{c}$, or the inhibition constant $K_{i}^{A}$. However, to avoid altering the dynamic response, the rate of the measurement flux $g_{c}$ is the prime target as this parameter does not affect the integral gain $G_{i}$ of the controller. If the goal is to change the dynamic response, (15) shows that the integral gain $G_{i}$ cannot be changed without changing the setpoint by altering only a single parameters. Both the rate of the z-flux $z_{c}$, and the inhibition constant $K_{i}^{A}$ affects not only the gain but also the setpoint. To change the gain without changing the setpoint one option is to adjust $z_{c}$ and $g_{c}$ by the same proportion.

If the flux is an enzyme catalyzed reaction, the rate can be changed by altering the enzyme's catalytic constant (turnover rate). This can be done in a static manner by genetically changing the enzyme (e.g. changing the enzyme with another related variant), or in a dynamic manner by introducing a reaction inhibitor of the non-competitive type [22], or by using a promotor to alter the level of transcription of the enzyme. For example ClpXP protease (a serine protease complex) from the bacteria E. coli have been expressed in yeast under control of a repressible promoter [26]. By adjusting the promotor in the incubation medium Grilly et al. [26] was able control the level of ClpXP protease which again controlled the degradation rate of a specific fluorescent protein.

\section{COMBINATION OF INFLOW AND OUTFLOW MOTIFS}

We will here show how the combination of one inflow and outflow controller motif can work together to control the concentration of a chemical species (controlled variable). Such pairwise antagonistic control, where one chemical species (e.g. 


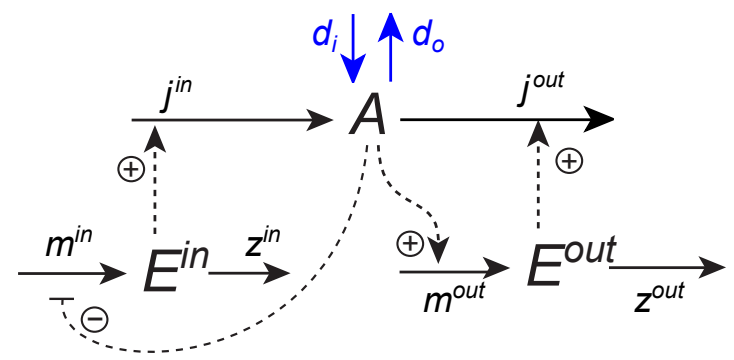

Fig. 6. Example system by combining an inflow controller of type 3 (left) and an outflow controller of type 5 (right).

a hormone) acts to increase the concentration of the controlled species while another chemical species acts to decrease it, is often seen in biophysical systems. One example is the blood calcium regulation by parathyroid hormone (increases calcium) and calcitonin hormone (decreases calcium). The reasons for why biological systems often have two controllers instead of just one is not completely understood [27]. Saunders and colleagues examined this question and introduced the theory of integral rein control [28], [29] in an attempt to explain the use of two controllers. By using our controller motifs we will here show how an antagonistic system can be explained and seen from an industrial viewpoint.

The system we consider contains two controller motifs, shown in Fig. 6, with individual setpoints that control the same variable, and hence, the system may appear over-specified. The two controllers however, have different operational behaviors. The right controller in Fig. 6 is an outflow controller (type 5) that works at relative high inflows of the controlled variable by removing excess of it. The controller to the left is an inflow controller (type 3 ) that works at high outflow of the controlled variable by adding sufficient amounts to the system.

The differential equations that describes the system are:

$$
\begin{aligned}
\dot{A}(t)= & d_{i}(t)-d_{c}(t) A(t)+f_{c}^{\text {in }} E^{\text {in }}(t) \\
& -f_{c}^{\text {out }} A(t) E^{\text {out }}(t) \\
\dot{E}^{\text {in }}(t)= & g_{c}^{\text {in }} \frac{K_{i}^{A}}{K_{i}^{A}+A(t)}-z_{c}^{\text {in }} \frac{E^{\text {in }}(t)}{K_{M}^{\text {in }}+E^{\text {in }}(t)} \\
\dot{E}^{\text {out }}(t)= & g_{c}^{\text {out }} A(t)-z_{c}^{\text {out }} \frac{E^{\text {out }}(t)}{K_{M}^{\text {out }}+E^{\text {out }}(t)}
\end{aligned}
$$

Both controllers have $g(\cdot)=1$ in their measurement fluxes and $z(\cdot)$ is a saturation type function of the level of $E^{i n}$ for the inflow motif and $E^{\text {out }}$ for the outflow motif (MichaelisMenten reaction kinetics [22]). The compensatory flux from the inflow controller has $f(\cdot)=1$, whereas the compensatory flux from the outflow controller has $f(\cdot)=A(t)$

The real setpoints of the two controller motifs will not be exactly the same as the theoretical setpoints shown in (10) and (13), because $z(\cdot)$ is dependent on the level of the controller variables. At low $K_{M}$ values however, the real setpoints and the theoretical setpoints will be close $\left(K_{M} \ll E\right)$.

Using terminology from control engineering, a tank based analogy (including valves and pumps) of the control problem is

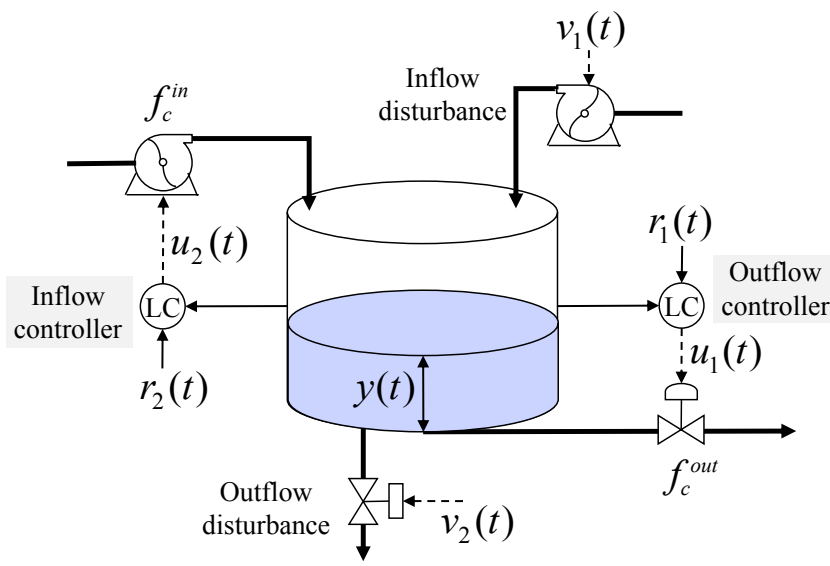

Fig. 7. Visualization of the control problem. See main text for definition of components.

shown in Fig. 7, where the variables and constants are renamed as follows:

1) $y(t)=A(t)$ as the tank level (controlled variable).

2) $u_{1}(t)=E^{\text {out }}(t)$ and $u_{2}(t)=E^{\text {in }}(t)$ as manipulated variables.

3) $v_{1}(t)=d_{i}(t)$ and $v_{2}(t)=d_{c}(t)$ as inflow and outflow disturbances.

4) $r_{1}(t)$ and $r_{2}(t)$ as the theoretical setpoints $A_{\text {set }}^{\text {out }}(t)$ and $A_{\text {set }}^{\text {in }}(t)$.

5) $f_{c}^{\text {in }}$ as the pump capacity constant.

6) $f_{c}^{\text {out }}$ as the outflow valve capacity constant.

For the sake of the argument we will assume that the outflow through the valves behaves linearly in the level of $\mathrm{A}(\mathrm{t})$.

The above shows the similarities between the biochemical motifs in Fig. 6 and a common control engineering problem as shown in Fig. 7.

This system functions properly when the setpoint of the inflow controller $A_{\text {set }}^{\text {in }}$ is lower or equal to the setpoint of the outflow controller $A_{\text {set }}^{\text {out }}$. If this is not the case the controller motifs will compete against each other, and as a consequence, there will be windup issues. The reason for this is that the inflow controller will add more and more of $A$ whereas the outflow controller will remove more and more of $A$ in order to track their respective setpoints.

Fig. 8 shows a simulation of this two controller system when $A_{\text {set }}^{\text {in }}<A_{\text {set }}^{\text {out }}$, showing how the inflow controller dominates during high outflows and how the outflow controller dominates during high inflow.

For the period $0<t<120$, the outflow disturbance $v_{2}$ dominates over the inflow disturbance $v_{1}$ (Fig. 8A), and thus the inflow controller is active (Fig. 8C) and adds more of $A$ to maintain its setpoint $r_{2}$.

When the disturbances are such that the output settles between the two setpoints, as in the period $120<t<180$, the output of both controller motifs are close to zero. The inflow motif is unactive because the level of $A$ is higher than the setpoint of this motif. The outflow motif is not yet active because its setpoint is higher than the level of $A$. 

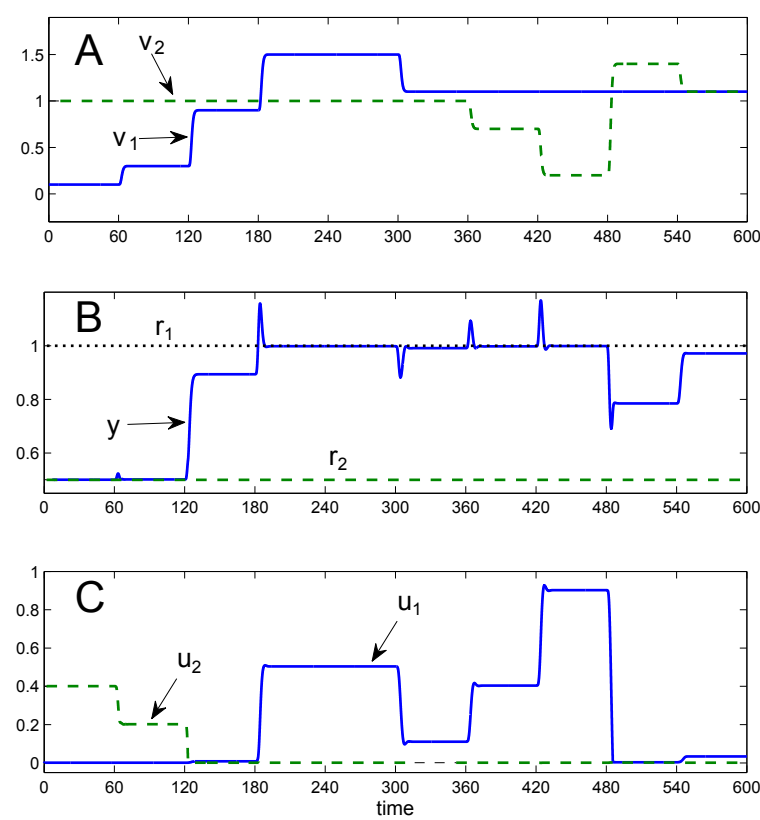

Fig. 8. Simulation demonstrating the disturbance rejection of a system with both inflow and outflow controller motifs. A. The system is perturbed by the disturbances in inflow $v_{1}$ and outflow $v_{2}$. B. The output $y$ is held at the inflow setpoint $r_{2}$ under dominating outflow $v_{2}$, and at the outflow setpoint $r_{1}$ under dominating inflow $v_{1}$. C. The level of the controller species $u_{1}$ and $u_{2}$ (manipulated variables). Model parameters in arbitrary units: $g_{c}^{i n}=1000$ $g_{c}^{\text {out }}=2.0, K_{M}^{i n}=K_{M}^{\text {out }}=K_{i}^{A}=0.001$, and $z_{c}^{\text {in }}=z_{c}^{\text {out }}=1.0$.

When the inflow disturbance $v_{1}$ dominates, as in the period $180<t<480$, the outflow motif is active and maintains the level of $A$ at its setpoint $r_{1}$.

\section{CONCLUDING REMARKS}

We have shown that reaction kinetic schemes of biophysical systems can be presented as control systems with setpoints, manipulated variables and controlled variables. An important property of these biochemical systems is that one can identify a controller species that behaves structurally similar to the integral control law from control engineering.

The similarities between biophysical systems and industrial control problems was illustrated by looking at a biophysical system consisting of two controller motifs (inflow and outflow), as structurally being the same problem as regulation of the level of fluid in a tank.

We emphasize that when looking to identify control structures in physiological experiments the type of motif (inflow/outflow 1-8) is a qualitative assessment, while the quantitative response of such control structures is a function of the reaction kinetics of the biochemical processes behind the motif.

\section{REFERENCES}

[1] G. M. Hughes, Homeostasis and Feedback Mechanisms. New York, NY, USA: Academic Press, 1964.

[2] N. Wiener, Cybernetics: or Control and Communication in the Animal and the Machine, 2nd ed. Cambridge, MA, USA: MIT Press, 1965.

[3] E. D. Sontag, "Molecular systems biology and control," Eur. J. Control, vol. 11, pp. 1-40, 2005.
[4] X. Y. Ni, T. Drengstig, and P. Ruoff, "The control of the controller: molecular mechanisms for robust perfect adaptation and temperature compensation," Biophys. J., vol. 97, no. 5, pp. 1244-53, Sep. 2009.

[5] N. Barkai and S. Leibler, "Robustness in simple biochemical networks," Nature, vol. 387, pp. 913-917, 1997.

[6] J. M. Carlson and J. Doyle, "Complexity and robustness," Proc. Natl. Acad. Sci. U.S.A., vol. 99 Suppl 1, pp. 2538-2545, Feb. 2002.

[7] J. Stelling, U. Sauer, Z. Szallasi, F. J. Doyle, and J. Doyle, "Robustness of cellular functions," Cell, vol. 118, no. 6, pp. 675-85, Sep. 2004.

[8] L. L. Langeley, Ed., Homeostasis Origins of the Concept. Stroudsburg, PA, USA: Dowden, Hutchinson \& Ross, 1973.

[9] S. J. Cooper, "From Claude Bernard to Walter Cannon. Emergence of the concept of homeostasis," Appetite, vol. 51, no. 3, pp. 419-27, Nov. 2008.

[10] H. El-Samad, J. P. Goff, and M. Khammash, "Calcium homeostasis and parturient hypocalcemia: an integral feedback perspective," J. Theor. Biol., vol. 214, no. 1, pp. 17-29, Jan. 2002.

[11] T. Drengstig, H. R. Ueda, and P. Ruoff, "Predicting perfect adaptation motifs in reaction kinetic networks," J. Phys. Chem. B, vol. 112, no. 51, pp. 16752-8, Dec. 2008.

[12] C. H. Hansen, R. G. Endres, and N. S. Wingreen, "Chemotaxis in Escherichia coli: a molecular model for robust precise adaptation," PLoS Comput. Biol., vol. 4, no. 1, pp. 14-27, Jan. 2008.

[13] W. Ma, A. Trusina, H. El-Samad, W. A. Lim, and C. Tang, "Defining network topologies that can achieve biochemical adaptation," Cell, vol. 138, no. 4, pp. 760-73, Aug. 2009.

[14] S. Skogestad and I. Postlethwaite, Multivariable Feedback Control, 2nd ed. Wiley, 2005.

[15] S. E. Brodie, B. W. Knight, and F. Ratliff, "The spatiotemporal transfer function of the Limulus lateral eye," J. Gen. Physiol., vol. 72, no. 2, pp. 167-202, Aug. 1978.

[16] T. Drengstig, I. Jolma, X. Ni, K. Thorsen, X. Xu, and P. Ruoff, "A basic set of homeostatic controller motifs," Biophys. J., vol. 103, no. 9, pp. 2000-2010, Nov. 2012.

[17] L. Kerkeb, I. Mukherjee, I. Chatterjee, B. Lahner, D. E. Salt, and E. L. Connolly, "Iron-induced turnover of the Arabidopsis IRT1 metal transporter requires lysine residues," Plant Physiol., vol. 146, no. 4, pp. 1964-73, Apr. 2008.

[18] M. S. LeBoff, D. Shoback, E. M. Brown, J. Thatcher, R. Leombruno, D. Beaudoin, M. Henry, R. Wilson, J. Pallotta, S. Marynick, J. Stock, and G. Leight, "Regulation of parathyroid hormone release and cytosolic calcium by extracellular calcium in dispersed and cultured bovine and pathological human parathyroid cells," J. Clin. Invest., vol. 75, no. 1, pp. 49-57, Jan. 1985.

[19] M. Matsuda, T.-A. Yamamoto, and M. Hirata, " $\mathrm{Ca}^{2+}$-dependent regulation of calcitonin gene expression by the transcriptional repressor DREAM," Endocrinology, vol. 147, no. 10, pp. 4608-17, Oct. 2006.

[20] J. Ang, S. Bagh, B. P. Ingalls, and D. R. McMillen, "Considerations for using integral feedback control to construct a perfectly adapting synthetic gene network." J. Theor. Biol., vol. 266, no. 4, pp. 723-38, Oct. 2010.

[21] S. Bagh, M. Mandal, J. Ang, and D. R. McMillen, “An active intracellular device to prevent lethal disease outcomes in virus-infected bacterial cells." Biotechnol. Bioeng., vol. 108, no. 3, pp. 645-654, Mar. 2011.

[22] A. Cornish-Bowden, Fundamentals of Enzyme Kinetics, 2nd ed. London, UK: Portland Press, 1995.

[23] J. Wilkie, M. A. Johnson, and R. Katebi, Control Engineering: An Introductory Course. New York, NY, USA: Palgrave, 2002.

[24] C. Cosentino and D. Bates, Feeback Control In Systems Biology. Boca Raton, FL, USA: CRC Press, 2012.

[25] S. A. Marshall, Introduction to Control Theory. London, UK: Macmillian Press, 1987.

[26] C. Grilly, J. Stricker, W. L. Pang, M. R. Bennett, and J. Hasty, “A synthetic gene network for tuning protein degradation in Saccharomyces cerevisiae." Mol. Syst. Biol., vol. 3, no. 127, p. 127, Jan. 2007.

[27] R. Sole and B. Goodwin, Signs of Life: How Complexity Pervades Biology. Basic Books, 2001.

[28] P. T. Saunders, J. H. Koeslag, and J. A. Wessels, "Integral rein control in physiology," J. Theor. Biol., vol. 194, pp. 163-173, Sep. 1998.

[29] —- "Integral rein control in physiology II: a general model," J. Theor Biol., vol. 206, no. 2, pp. 211-20, Sep. 2000. 